\section{Preceptoria na Residência de Medicina de Família e Comunidade da Universidade de São Paulo: políticas e experiências}

\author{
Preceptorship in the Family Medicine Residence of Universidade \\ de São Paulo: policies and experiences \\ Preceptoría en la Residencia de Medicina Familiar y Comunitaria \\ de la Universidade de São Paulo: políticas y experiencias
}

\section{Resumo}

Introdução: A atividade de preceptoria dentro da formação médica tem sido globalmente estabelecida como modelo para o processo docente na área e existem diversas formas e experiências de organização. O Programa de Residência Médica (PRM) em Medicina de Família e Comunidade (MFC) da Faculdade de Medicina da Universidade de São Paulo (FMUSP) apresenta um modelo de preceptoria incomum, composto por preceptores do programa de residência médica e por preceptores dos residentes (tutores), cada um com especificidades próprias e complementares. Objetivo: Nosso objetivo neste relato de experiência foi compartilhar as políticas institucionais e experiências de preceptoria em nosso programa de residência médica, bem como apontar as múltiplas adversidades existentes e possíveis formas de enfrentamento. Resultados: Atingir o nível de excelência no desenvolvimento das competências necessárias para a formação do especialista em MFC, em situações adversas de valorização da especialidade e do corpo docente, é uma tarefa árdua. Neste difícil desafio de conduzir o PRM e garantir o desenvolvimento das competências almejadas com qualidade, o papel do preceptor do programa de residência médica e do preceptor do residente (tutor) são fundamentais. Conclusão: Frente aos desafios apontados, observa-se grande desgaste dos tutores, que voluntariamente desempenham as atividades de ensino mesmo atuando em sobrecarga assistencial. A falta de reconhecimento, escasso apoio institucional e da gestão administrativa são fatores que oferecem obstáculos ao melhor desenvolvimento e ampliação do programa de residência.

Palavras-chave: Preceptoria; Internato e Residência; Medicina de Família e Comunidade; Atenção Primária à Saúde; Educação Médica
Ana Paiva Garcia

Luiza Magalhães Cadioli

Ademir Lopes Júnior

Gustavo Gusso

José Benedito Ramos Valladão Júnior

Faculdade de Medicina da Universidade de São Paulo (FMUSP). São Paulo, SP, Brasil. anapaiva02@yahoo.com.br (Autora correspondente); luiza.cadioli@gmail.com; ad.lopesjunior@gmail.com; gusso.gustavo@ gmail.com; jose.valladao@usp.br

Fonte de financiamento: declaram não haver. Parecer CEP: não se aplica. Conflito de interesses: declaram não haver. Procedência e revisão por pares: revisado por pares.

Como citar: Garcia AP, Cadioli LM, Lopes Júnior A, Gusso G, Valladão Júnior JBR. Preceptoria na Residência de Medicina de Família e Comunidade da Universidade de São Paulo: políticas e experiências. Rev Bras Med Fam Comunidade. 2018;13(40):1-8. http://dx.doi.org/10.5712/rbmfc13(40)1610
Recebido em: 30/08/2017.

Aprovado em: 09/12/2017. 


\begin{abstract}
Introduction: The preceptorship activity within medical education has been globally established as a model for the teaching process in the area and there are several forms and experiences of organization. The Medical Residency Program in Family Medicine of Universidade de São Paulo presents an unusual model of preceptors, composed of preceptors of the medical residency program, each one with its own and complementary specificities. Objectives: Our goal in this experience report was to share the institutional policies and preceptory experiences in our medical residency program, as well as to point out the multiple adversities and possible forms of coping. Results: Achieving the level of excellence in developing the skills needed to train the Family Medicine specialist, in adverse situations of appreciation of the specialty and the teaching staff, is an arduous task. In this difficult challenge of conducting the Medical Residency Program and ensuring the development of quality skills, the role of the preceptor of the medical residency program and the resident's tutor is crucial. Conclusion: Faced with the challenges pointed out, there is great wear and tear on the tutors, who voluntarily perform the teaching activities even when acting on welfare overload. The lack of recognition, scarcity of institutional support and administrative management are factors that hinder the better development and expansion of the residency program.
\end{abstract}

Keywords: Preceptorship; Internship and Residency; Family Practice; Primary Health Care; Education, Medical

\title{
Resumen
}

Introducción: La actividad de preceptoría dentro de la formación médica ha sido globalmente establecida como modelo para el proceso docente en el área y existen diversas formas y experiencias de organización. El Programa de Residencia Médica (PRM) en Medicina Familiar y Comunitaria (MFyC) de la Universidade de São Paulo presenta un modelo de preceptoria inusual, compuesto por preceptores del programa de residencia médica y por preceptores de los residentes (tutores), cada uno con especificidades propias y complementarias. Objetivo: Nuestro objetivo en este relato de experiencia fue compartir las políticas institucionales y experiencias de preceptoría en nuestro programa de residencia médica, así como apuntar las múltiples adversidades existentes y posibles formas de enfrentamiento. Resultados: Alcanzar el nivel de excelencia en el desarrollo de las competencias necesarias para la formación del especialista en Medicina Familiar, en situaciones adversas de valorización de la especialidad y del cuerpo docente, es una tarea ardua. En este difícil desafío de conducir el Programa de Residencia Médica y garantizar el desarrollo de las competencias anheladas con calidad, el papel del preceptor del programa de residencia médica y del preceptor del residente (tutor) son fundamentales. Conclusión: Ante los desafíos señalados, hay gran desgaste de los tutores, que voluntariamente desempeñan las actividades de enseñanza incluso actuando en sobrecarga asistencial. La falta de reconocimiento, escaso apoyo institucional y de la gestión administrativa son factores que dificultan el desarrollo y la expansión del programa de residencia.

Palabras clave: Preceptoría; Internado y Residencia; Medicina Familiar y Comunitaria; Atención Primaria de Salud; Educación Médica

"Quando vivemos a autenticidade exigida pela prática de ensinar-aprender participamos de uma experiência total, diretiva, política, ideológica, gnosiológica, pedagógica, estética, ética, em que a boniteza deve achar-se de mãos dadas com a decência e com a seriedade"

Paulo Freire ${ }^{1}$

\section{Introdução}

A preceptoria tem sido estabelecida como modelo formador para a residência médica por integrar atividades didáticas e práticas em serviço. Todavia, há grande variabilidade de significados, funções e atividades vinculadas ao cargo de preceptor de acordo com as diferenças entre instituições de ensino médico. ${ }^{2}$

O Programa de Residência Médica (PRM) em Medicina de Família e Comunidade (MFC) da Faculdade de Medicina da Universidade de São Paulo (FMUSP) possui, caracteristicamente, desde sua formação e, em consonância com outros PRMs da instituição, a figura do preceptor do programa de residência médica. Além disso, também existem os preceptores dos residentes (tutores). Estes dois atores desempenham diferentes papéis, com complementaridades e interfaces envolvidas no processo de docência e organização da residência médica. 
As funções dos preceptores do programa de residência médica incidem na organização e gestão de todas as atividades correspondentes à formação dos médicos-residentes e pelo controle do cumprimento dos objetivos do programa, conforme o Manual de Competências da Residência. ${ }^{3} \mathrm{O}$ preceptor do residente (tutor) é um médico especialista em MFC que atua como médico contratado de uma equipe de Estratégia de Saúde da Família (ESF) em uma Unidade Básica de Saúde (UBS) utilizada como cenário de prática do PRM durante o Estágio Longitudinal de Atenção Primária da residência.

\section{Desenvolvimento}

Esse artigo pretende apresentar dois tipos de preceptoria realizada no Programa de Residência Médica em Medicina de Família e Comunidade (PRM-MFC) da Universidade de São Paulo (USP) e os desafios para sua consolidação.

\section{Programa de Residência}

O PRM-MFC foi iniciado em 2005 e atualmente tem 10 residentes por ano. Os residentes estão distribuídos em 5 unidades de atenção primária à saúde da Região Oeste, quatro delas unidades básicas de saúde (UBS) da microrregião do bairro do Butantã, atualmente geridas pela Associação Paulista para o Desenvolvimento da Medicina (SPDM), e um Centro de Saúde Escola pertencente à própria Universidade de São Paulo (CSE Butantã). Os estágios nos níveis secundário e terciário de atenção representam em torno de $20 \%$ da carga horária da residência e são realizados principalmente no Hospital Universitário (HU-USP), e no Hospital das Clínicas (HC), que é uma autarquia estadual vinculada à Secretaria de Estado da Saúde para fins de coordenação administrativa e associada à Faculdade de Medicina da USP para fins de ensino.

\section{Preceptor do Programa de Residência Médica}

Anualmente, são oferecidas duas vagas para a preceptoria do PRM-MFC através de processo seletivo organizado pela coordenação do programa. O cargo é rotativo, existindo a possibilidade de o preceptor realizar novamente o processo seletivo e obter a prorrogação do cargo de preceptoria, no máximo, por mais um ano. Dentre os pré-requisitos, o candidato ao cargo deverá ter concluído residência médica na especialidade dentro dos últimos três anos. O objetivo é que, justamente, participem como preceptores do programa de residência médica em apoio ao coordenador do PRM, os médicos especialistas em MFC recém-egressos. O intuito é que o cargo seja ocupado por quem está mais próximo da realidade da residência e conheça os pontos positivos, negativos, problemas dos estágios e do cotidiano dos residentes.

As atividades específicas realizadas pelo preceptor do programa de residência médica são divididas em sete vertentes de funções:

- Planejamento e organização: planejar e organizar calendário de aulas e estágios anualmente em função dos recursos disponíveis, do número de tutores e residentes e supervisionar sua posterior elaboração e prática pelos serviços hospitalares, ambulatórios e unidades de saúde correspondentes; prover estrutura para garantir a realização de outras atividades, como participação em campanhas de vacina, espaços de participação popular (como conselho gestor, conselhos distritais); fomentar e prover suporte ao residente para planejamento e execução de 
atividade de grupo na unidade de saúde durante a residência; realizar avaliação e orientações junto aos residentes e tutores para o aperfeiçoamento; organização de cursos, seminários ou workshops que se considerem de interesse para o desenvolvimento de habilidades práticas úteis na especialidade de Medicina de Família; organizar a recepção e programação de estagiários; organizar os espaços de reunião geral da residência, sendo responsável pelo convite de professores, cronograma, envio de referências, reserva de espaço e divulgação; realizar reuniões periódicas com os tutores; participar dos processos seletivos de novos residentes; manter atualizados o manual da residência, a carta de estágios eletivos e o currículo de competências do PRM.

- Atividades de supervisão e ensino: realizar cursos e módulos conforme a necessidade do programa; estar disponível, estimular e realizar procedimentos junto aos residentes nas unidades de saúde; dar apoio aos residentes na preparação de sessões clínicas e discussões de artigos; orientar e auxiliar os residentes quanto a solicitações de estágios externos e realização de estágios optativos; reunir informação detalhada sobre todas as atividades que cumpram cada residente; realizar discussões clínicas, atividades de observação de consultas e espaços de escuta ao residente; propor leituras e discussões conforme avaliação das necessidades formativas individualizadas de cada residente; apoiar os tutores nas dificuldades apresentadas durante o processo de docência; estimular a formação dos tutores e procurar organizar atividades de aperfeiçoamento.

- Monitorização das atividades docentes: detectar e analisar deficiências formativas, realizar propostas de melhoria e apoio técnico às unidades de saúde com residentes; dar apoio e estímulo ao trabalho dos tutores do programa da especialidade; realizar comunicação com tutores hospitalares de outras especialidades longitudinalmente de forma a garantir o bom andamento dos estágios; detecção e análise de deficiências formativas no hospital com relação à Medicina de Família e Comunidade; proposta de medidas de melhoria na organização docente hospitalar.

- Pesquisa: estimular e apoiar os projetos de pesquisas dos residentes, fornecendo auxílio na busca de orientadores, pesquisa bibliográfica e dúvidas; garantir a estrutura e supervisão de formação específica em pesquisa científica na forma de estágio ou workshops para prover o conhecimento necessário e o suporte aos diferentes trabalhos de pesquisa que realizem os médicos residentes durante seu período de formação.

- Avaliação: preparar questões para prova dos residentes que deverá ser realizada semestralmente, aplicar e corrigir as provas, bem como, fornecer espaço para discussão de respostas e recursos aos residentes; aplicar ferramenta de avaliação de consultas dos residentes através do COT (Consultation Observation Tool) semestralmente com a distribuição dos residentes conforme tutores que os avaliarão; garantir a realização das demais avaliações dos residentes (estágio longitudinal em APS, estágios hospitalares e externos, apresentação de artigo e de caso clínico), submeter todos os tutores e estágios à avaliação dos residentes; realizar exposição dos resultados das avaliações nos fóruns pertinentes anualmente ao final do período letivo; entregar trimestralmente a avaliação de todos os residentes à Comissão de Residência Médica (COREME). 
- Responsabilidade com a instituição: realizar aulas ou apresentações sobre a especialidade conforme solicitação de outros programas de residência ligados à instituição; colaboração com a Universidade em outras atividades e espaços da graduação a que for solicitado; emissão de relatórios e informes às autoridades administrativas conforme solicitação da coordenação do PRM e da COREME.

- Comunicação: manter-se disponível para responder e-mails, mensagens de celular e telefonemas de residentes, tutores, coordenação; manter grupos de comunicação em celular com os diferentes grupos componentes da residência visando facilitar a comunicação.

A atividade do preceptor de programa é regulamentada pela COREME da Faculdade de Medicina da USP. Ele recebe uma bolsa da FMUSP equivalente ao dobro do valor vigente da bolsa recebida pelos residentes, com carga-horária semanal de 40 horas. É, conforme descrito acima, corresponsável por organizar e coordenar as atividades didáticas junto ao coordenador, oferecendo suporte a todos os residentes e tutores. Vale ressaltar que não faz parte de suas atribuições a assistência direta isolada. No entanto, poderá desenvolver atividades de assistência didática voltada ao ensino, como recurso pedagógico.

\section{Preceptor do Residente (Tutor)}

A maior parte das atividades do PRM-MFC são realizadas no Estágio Longitudinal de Atenção Primária, que ocupa $65 \%$ da carga horária durante os dois anos de residência. Nesse estágio, o residente faz parte de uma equipe da Estratégia de Saúde da Família (ESF) em que existe um especialista em MFC em atividade como médico da equipe e que será o seu respectivo tutor. Esta é uma característica distintiva deste modelo para o de muitas outras residências de MFC no Brasil. Por ser o médico da equipe em que o residente está inserido e quem está mais próximo do residente ao longo dos dois anos, o tutor é um elo fundamental na formação do residente em MFC.

Os casos atendidos pelos residentes são, majoritariamente, discutidos com o seu tutor pessoal (médico da própria equipe), uma vez que conhecem os pacientes, mas é também estimulado o modelo de tutoria cruzada, quando o residente de uma equipe também discute com os tutores de outra, por meio de uma escala de discussão entre os médicos tutores nas respectivas UBSs.

O papel do preceptor dos residentes envolve: auxiliar no aprendizado de conceitos teóricos direcionados à prática, supervisionar a prática do residente, ser modelo de valores, atitudes e ética profissional; auxiliar na identificação de qualidades a serem ressaltadas e ou corrigidas; compartilhar experiências acerca da profissão e formas de lidar com as inseguranças e frustrações. ${ }^{2,4}$

Para o adequado desempenho de tais papéis na formação do médico-residente, recomenda-se que o tutor busque incorporar algumas funções nucleares em sua atividade docente (Quadro 1).

O tutor deve, portanto, fornecer o exemplo profissional, compartilhar conhecimentos e inquietações, dividir o trabalho e responsabilidades da equipe progressivamente, adaptar e flexibilizar conteúdos e métodos conforme as necessidades de seu residente. Deverá ter uma relação mutuamente colaborativa centrada no aprendizado. Não necessariamente de amizade, nem impessoal ou autoritária. Deverá existir uma relação dialógica, interpessoal, horizontal, de esperança e respeito, construída e personalizada às expectativas e necessidades de cada residente. 
Quadro 1. Funções nucleares do preceptor do residente (tutor).

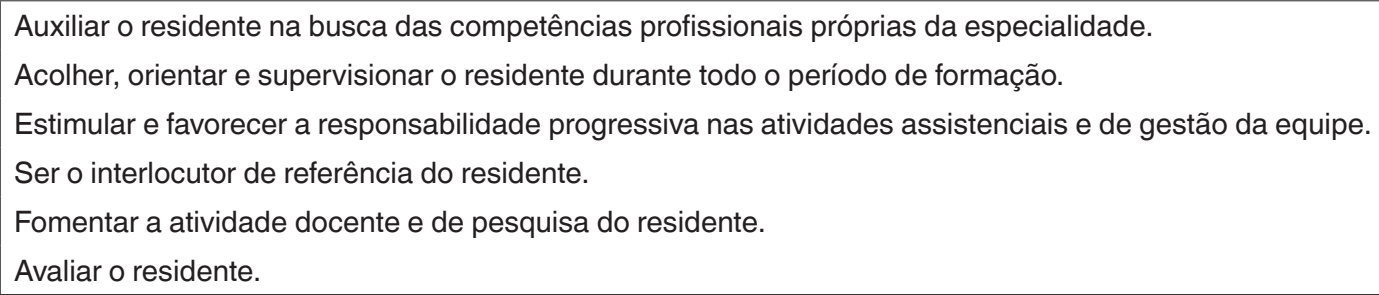

A preceptoria deve ser centrada no residente e orientada a fim de instrumentalizá-lo para a resolução de problemas da prática profissional com progressiva autonomia. ${ }^{5,6}$

Inicialmente, a contratação dos preceptores dos residentes foi feita pela Fundação Faculdade de Medicina (FFM), que é uma Fundação de Apoio à FUMSP e ao Hospital das Clínicas (HCFMUSP). A partir de 2008, a FFM tornou-se uma OS e fundou o Projeto Região Oeste (PRO), que passou a gerir as unidades básicas da região do Butantã, cenário de prática da residência MFC da USP. Esse projeto manteve-se até 2016, quando outra OS, não vinculada à FMUSP, passou a gerir a região.

A contratação e pagamento de todos os profissionais da região pela Fundação Faculdade de Medicina, via PRO, entre eles os médicos tutores da residência, mascarava a total ausência de vínculo entre a preceptoria dos residentes (tutores) e o órgão que oferece esta residência. Com o processo de transição de OS e a saída do PRO, esta situação se tornou mais evidente.

Atualmente, o vínculo empregatício e a remuneração do tutor das quatro UBSs da prefeitura são realizados pela SPDM com jornadas de 20, 30 ou 40h semanais. Esses tutores não possuem nenhum tipo de ligação formal com a FMUSP, exercendo sua função de preceptoria de forma voluntária, sem remuneração ou qualquer tipo de incentivo, ao contrário do recomendado pelo guia para programas de pós-graduação em medicina de família do WONCA. ${ }^{7}$

Não há, também, por parte da secretaria municipal de saúde, redução de metas assistenciais ou previsão de carga horária diferenciada para médicos tutores. A OS contratante disponibiliza apenas o período da reunião geral do PRM-MFC (que dura 1 hora e meia) e $1 \mathrm{~h}$ para os seminários dos residentes, ambos uma vez por semana. Na dependência da gerência da unidade, essas atividades são consideradas como Educação Permanente e se estendem a todos médicos do serviço, exercendo eles o papel de tutor ou não. Mas isso exige um acordo com o gerente, que pode não acontecer. A possibilidade de jornadas de trabalho de 20 e 30h, implantadas em 2016, têm sido atrativas para manter alguns tutores vinculados ao serviço.

Por não haver processo seletivo para ser tutor ou vínculo oficial institucional, este cargo geralmente é ocupado por algum médico com uma aproximação afetiva com a residência ou com interesse pelo ensino, como ex-residentes. Apesar disso, temos conseguido que todos os tutores tenham título de especialista em MFC ou residência em MFC.

\section{Desafios e Propostas}

Cientes das fragilidades e potencialidades desses modelos complementares de preceptoria e, visando a consolidação, a estabilidade e a expansão desse programa de residência em MFC, propõe-se: 
1. Reconhecimento institucional das atividades da tutoria pela FMUSP, por meio de certificados oficiais pelo exercício de preceptoria;

2. Acesso à rede de internet da FMUSP via VPN (que viabiliza o acesso a revistas cientificas eletrônicas) para os tutores, bem como incentivo a cursos de formação de tutores, como, por exemplo os da EURACT (European Academy of Teachers in General Practice);

3. Redução das metas assistenciais e do tamanho da população das equipes da ESF para viabilizar períodos para atividades de educação permanente e pós-graduação para os tutores;

4. Previsão das atividades de ensino na jornada de trabalho;

5. Flexibilidade na jornada de trabalho para que possa ser compatível com as atividades assistenciais e de ensino do PRMMFC;

6. Complementação financeira ou outros incentivos pelas atividades de preceptoria;

7. Implantação do Contrato Organizativo de Ação Pública Ensino-Saúde (COAPES) ${ }^{8}$ visando reunir as regras, contrapartidas e demais compromissos pactuados entre as instituições de ensino e os gestores municipais e estaduais do SUS;

8. Contratação de médicos de família que tenham interesse no ensino e na participação do PRM-MFC em todas as equipes das UBS ligadas a residência médica;

9. Maior integração entre tutores e residentes com alunos da graduação, de forma a apresentar a prática clínica e as habilidades específicas da MFC desde o início da formação. Isso corrobora a implementação das Diretrizes Curriculares do Curso de Graduação de Medicina, com ênfase na formação generalista, de base humanista cujas habilidades sejam direcionadas a responder às necessidades de saúde da população, das comunidades e do Sistema de Saúde.

\section{Considerações finais}

A divisão de dois atores de diferentes características e atividades dentro do processo de preceptoria centrado no residente do Programa de Residência Médica (PRM) em Medicina de Família e Comunidade (MFC) da Faculdade de Medicina da USP (FMUSP) é algo distinto da maioria dos programas de residência médica.

As especificidades da instituição tornaram importante a conformação destes dois diferentes papéis para garantia do respeito a todas as necessidades de seus programas formativos e o alcance às competências almejadas.

O preceptor do programa de residência se dedica a ser o guardião de todo o programa de residência médica em suporte ao coordenador, acumulando funções administrativas, de ensino e avaliação, garantindo, assim, a execução de todas as prerrogativas docentes definidas.

O preceptor do residente (tutor) desempenha o processo docente específica e diretamente relacionado aos residentes sob sua supervisão. Desta forma, é premente a necessidade de políticas de valorização desses profissionais, objetivando sua fixação no PRM-MFC para a ampliação do número de vagas da residência e maior contribuição acadêmica e científica no cenário da atenção primária na USP e no Brasil. 
As interfaces e complementaridades destes modelos de preceptoria têm tido bons resultados dentro de nossa experiência por produzirem um sólido programa docente junto a uma supervisão de excelência propiciada pela atuação conjunta destes diferentes preceptores. Por um lado, a figura do preceptor do programa, permite suporte aos preceptores dos residentes (tutores), que atuam em situações adversas, com sobrecarga de atividade assistencial e atividade docente voluntária, garantindo a organização didática da residência e a integração das atividades de ensino entre as várias UBSs e cenários de aprendizagem. Por outro, estando os tutores na assistência, os residentes são protegidos em parte da pressão assistencial da equipe, reiterando seu espaço de formação.

A qualidade da preceptoria do PRM e dos tutores é um dos pontos fortes do PRM-MFC da FMUSP. Entretanto, para a consolidação e ampliação do programa, é urgente e imprescindível a implantação de políticas para valorização desses profissionais, objetivando sua fixação e vinculação ao PRM-MFC. Podemos considerar esse o nó crítico para a ampliação do número de vagas de residência em MFC e que não foi enfrentado desde o início do PRM-MFC da Universidade de São Paulo.

\section{Referências}

1. Freire P. Pedagogia da Autonomia: saberes necessários à prática educativa. 25a ed. São Paulo: Paz e Terra; 1996.54 p [Internet]. [acesso 2017 Dez 4]. Disponível em: http://forumeja.org.br/files/Autonomia.pdf

2. Botti SHO, Rego S. Preceptor, supervisor, tutor e mentor: quais são seus papéis? Rev Bras Educ Med. 2008;32(3):363-73 [Internet]. [acesso 2017 Dez 4]. DOI: http://dx.doi.org/10.1590/S0100-55022008000300011

3. Valladão Júnior JBR, Gusso G, Olmos RD. Medicina de família e comunidade - Série Manual do Médico-Residente do Hospital das Clínicas da Faculdade de Medicina da Universidade de São Paulo. São Paulo: Atheneu;2017. 720 p.

4. Bain L. Preceptorship: a review of the literature. J Adv Nurs. 1996;24(1):104-7 [Internet]. [acesso 2017 Dez 4]. DOI: http://dx.doi. org/10.1046/j.1365-2648.1996.15714.x

5. Maudsley G. Roles and responsibilities of the problem based learning tutor in the undergraduate medical curriculum. BMJ [Internet]. 1999;318(7184):657-61. [acesso 2017 Dez 4]. Disponível em: http://www.bmj.com/cgi/doi/10.1136/bmj.318.7184.657

6. Freire P. Educação Como Prática da Liberdade. 1a ed. Rio de Janeiro: Paz e Terra; 1967. 150 p [Internet]. [acesso 2017 Dez 4]. Disponível em: http://www.dhnet.org.br/direitos/militantes/paulofreire/livro_freire_educacao_pratica_liberdade.pdf

7. WONCA working party on education. WONCA standards for postgraduate family medicine education. $2013.17 \mathrm{p}$ [Internet]. [acesso 2018 Fev 6]. Disponível em:http://www.globalfamilydoctor.com/site/DefaultSite/filesystem/documents/Groups/Education/WONCA\%20ME\%20 stds_edit\%20for\%20web_250714.pdf

8. Brasil. Ministério da Saúde. Portaria Interministerial № 1.127, de 04 de agosto de 2015. Institui as diretrizes para a celebração dos Contratos Organizativos de Ação Pública Ensino-Saúde (COAPES), para o fortalecimento da integração entre ensino, serviços e comunidade no âmbito do Sistema Único de Saúde (SUS). Brasília: Ministério da Saúde; 2015 [Internet]. [acesso 2017 Ago 26]. Disponível em: http://portalarquivos.saude.gov.br/images/pdf/2015/outubro/23/COAPES-PORTARIA-INTERMINISTERIAL-N1.127\%20-DE-04\%20 DE-AGOSTO-DE-2015.pdf 